\title{
Hydrogenated amorphous silicon multi-SOI waveguide modulator with low voltage-length product
}

\author{
Sandro Rao ${ }^{a, *}$, Giuseppe Coppola ${ }^{b}$, Mariano A. Gioffrè ${ }^{b}$, Francesco G. Della Corte ${ }^{a}$ \\ a Università degli Studi “Mediterranea” di Reggio Calabria, Department of Information Science, Mathematics, Electronics and Transportations (DIMET), Via Graziella Loc. \\ Feo di Vito, 89133 Reggio Calabria, Italy \\ ${ }^{\mathrm{b}}$ Institute for Microelectronics and Microsystems_Consiglio Nazionale delle Ricerche (IMM-CNR)—Unit of Napoli, Via Castellino, 111, 80132 Napoli, Italy
}

\section{A R T I C L E I N F O}

Article history:

Received 5 May 2012

Received in revised form

25 June 2012

Accepted 2 July 2012

Available online 23 July 2012

Keywords:

Amorphous materials

Electro-optic modulation

Integrated optics

\begin{abstract}
A B S T R A C T
Electrically induced phase modulation is characterized for the first time in a waveguide-integrated Fabry-Perot resonating cavity based both on an index- and conductivity- high-contrast amorphous silicon/amorphous silicon carbide (a-Si:H/a-SiC:H) multistack. The device consists of a single mode a-Si:H rib waveguide containing three insulating thin layers of a-SiC:H embedded within the core thickness. The effective refractive index change, $\Delta n_{\text {eff }}$, at the wavelength of $\lambda=1.55 \mu \mathrm{m}$ is achieved through the application of an electric field across the stack which induces carrier accumulation at all the a-Si:H/a-SiC:H interfaces, resulting in turn in a high interaction between the optical beam and the accumulation layers. This configuration allows to obtain a $V_{\pi} \times L_{\pi}$ product of about $5.9 \mathrm{~V} \mathrm{~cm}$, not far from what observed in high performing electro-optical modulators in crystalline silicon.
\end{abstract}

(c) 2012 Elsevier Ltd. All rights reserved.

\section{Introduction}

The realization of on-chip optical interconnects relies on the ability to integrate micro-optical devices with microelectronics. High performing all-silicon electro-optical modulators have been recently proposed and realized exploiting the free-carrier density variation to control the optical index of the device. The carrier concentration change is induced either by direct electrical injection [1] or by depletion [2]. Recently, hydrogenated amorphous silicon $(\mathrm{a}-\mathrm{Si}: \mathrm{H})$, deposited using the CMOS-compatible technique of low temperature $\left(120-300{ }^{\circ} \mathrm{C}\right)$ plasma-enhanced chemical vapour deposition (LT-PECVD), has also emerged as a useful material for enabling the back-end integration of passive and active optical components [3-7]. The low temperature process enables the substantial independency of the CMOS layer and the photonic layer, which may take place virtually in two distinct technological facilities, with no impact at all on the circuit design. On the other hand, a-Si:H technology could allow the direct fabrication of a photonic layer above a CMOS microchip as a low cost post-processing phase. Moreover, amorphous materials can be easily deposited with a bandgap tailored to cover a wide energy spectrum and therefore with a refractive index varying within a given range [8]. With these aims, the well established a-Si:H deposition technique has been used to realize optoelectronic devices based on a multistack configuration, which can be

\footnotetext{
* Corresponding author. Tel.: +39 0965875284; fax: + 390965875463.

E-mail address: sandro.rao@unirc.it (S. Rao).
}

exploited for enhancing the free-carrier effects through a more homogeneous distribution of the excess carriers across the waveguide core thickness, as demonstrated in multi-SOI [9] and a-Si:H devices [5,10-12]. In particular, in Ref. [10] a multistack structure composed of six a-Si:H-based bi-layers were explored for enhancing electro-optical modulation effects in an integrated optical modulator. On the other hand, in Ref. [7] we reported the first experimental results of an effective refractive index variation obtained through an electrically induced carrier depletion in an as-deposited a-Si:H p-i-n diode-based waveguiding device. In this paper, we combine both our previous studies to realize a three bi-layers interferometric modulator, demonstrating the advantages of this approach with respect to the electroabsorption approach.

\section{Device structure and fabrication}

A 3D schematic structure of the designed waveguide is shown in Fig. 1. The device consists of the stack of three bi-layers, each composed of a $t_{\mathrm{a}-\mathrm{Si}: \mathrm{H}}=300-\mathrm{nm}$-thick a-Si:H layer $\left(n_{\mathrm{a}-\mathrm{Si}: \mathrm{H}}=3.58\right.$, $\rho_{\text {a-Si:H }} \sim 10^{8} \Omega \mathrm{cm}$, band gap of $\sim 1.8 \mathrm{eV}$ ) and a $40 \mathrm{~nm}$-thick a-SiC:H layer $\left(n_{\mathrm{a}-\mathrm{SiC}: \mathrm{H}}=2.93, \rho_{\mathrm{a}-\mathrm{SiC}: \mathrm{H}} \sim 10^{12} \Omega \mathrm{cm}\right.$, band gap of $\sim 2.1 \mathrm{eV}$ ), deposited on a doped crystalline silicon (c-Si) substrate $\left(\rho_{\mathrm{c}-\mathrm{Si}}=0.001 \Omega \mathrm{cm}\right) . \rho_{\text {material }}$ is the resistivity, related to the doping level, of the material layer. The substrate and the $1.02 \mu \mathrm{m}$-thick stack are separated by a $1.1-\mu \mathrm{m}$-thick $\mathrm{SiO}_{2}$ low refractive index $\left(n_{\mathrm{SiO}_{2}}=1.45\right)$ cladding layer ensuring a good vertical optical confinement. 
Technological flowchart is very similar to that reported in our previous papers (Refs. $[5,7,10]$ ), however, in the following the main steps are described. The device fabrication begins with the plasma enhanced chemical vapour deposition of the $1.1 \mu \mathrm{m}$ silicon oxide on a silicon substrate. After deposition, several through circular hole vias, with a diameter of $100 \mu \mathrm{m}$ are opened by an HF wet etching of $\mathrm{SiO}_{2}$. These holes allow the electrical continuity between the substrate and the first a-Si:H layer and are positioned $200 \mu \mathrm{m}$ far from the waveguide centre in order not to perturb the optical field propagation into the waveguide. The first a-Si:H layer is then deposited from the plasma-assisted decomposition of $\mathrm{SiH}_{4}$ at a flow rate of $20 \mathrm{sccm}$ (standard cubic centimetres per minute) and an RF power $P_{R F}=4 \mathrm{~W}$. This layer also completely fills the circular hole vias. Subsequently, the a-SiC: $\mathrm{H}$ layer is deposited from a gaseous mixture of $\mathrm{SiH}_{4}$ and $\mathrm{CH}_{4}(25$ and $35 \mathrm{sccm}$ respectively, $P_{R F}=4 \mathrm{~W}$ ). The additional two pairs of a-Si:H/a-SiC:H are laid using the same PECVD process parameters reported above. All deposition steps are performed at a temperature of $200{ }^{\circ} \mathrm{C}$. Finally, the 100-nm-thick ZnO:Al transparent conductive oxide (TCO) is deposited by magnetron co-sputtering of $\mathrm{ZnO}$ and $\mathrm{Al}$ targets, at $25^{\circ} \mathrm{C}$

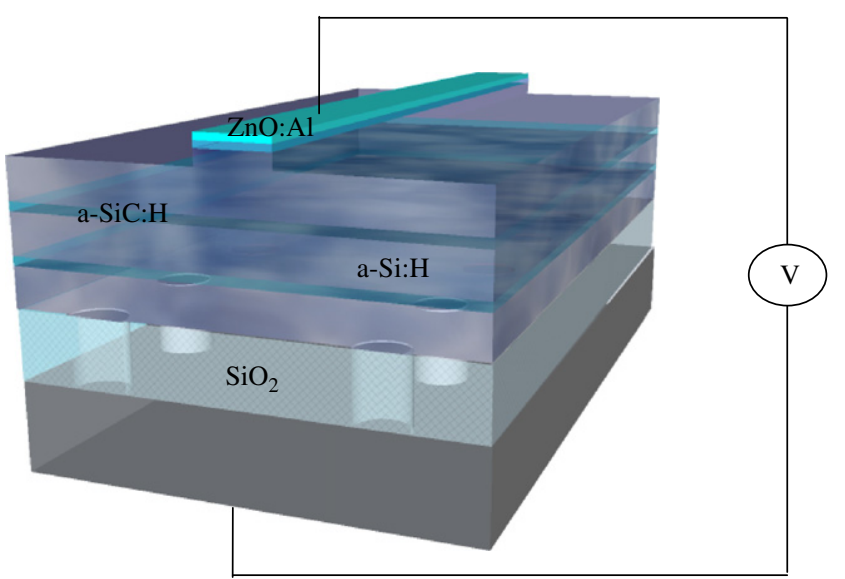

Fig. 1. 3D not to scale representation of the multilayer rib-waveguide. Small viaholes are present through the $\mathrm{SiO}_{2}$ cladding layer, on both sides of the rib waveguide, in order to ensure the electrical continuity between the highly doped silicon substrate and the first a-Si:H layer. substrate temperature. At the end of the deposition sequence, the fingerprint of the hole vias is clearly visible on the surface. However, as aforesaid, these holes do not affect the waveguide propagation characteristics due to their distance from the actual waveguiding region.

Standard technological processes have been used, i.e. optical lithography and reactive ion etching (RIE), to realise the rib waveguide with the TCO top contact (see Ref. [5]). Optical simulations, carried out by means of BeamPROP tool provided by RSoft [13], show that the designed waveguide supports only one mode for the TE polarization once a $4-\mu \mathrm{m}$-wide and $160-\mathrm{nm}$ high rib is defined. Due to the waveguide geometry, the TM mode exhibits high losses [14] and low modulation efficiency [15]; therefore only the TE-like polarization will be considered hereafter.

In order to exploit the effective refractive index variation for the optical modulation, Fabry-Perot (FP) cavities were obtained from the rib waveguide by defining two deep trenches spaced by $50 \mu \mathrm{m}$ as shown in Fig. 2. The 5- $\mu \mathrm{m}$-wide trenches are fabricated by a RIE process performed using a mixture of $20 \mathrm{sccm}$ of $\mathrm{SF}_{6}$ and $20 \mathrm{sccm}$ of $\mathrm{O}_{2}$ with an $\mathrm{RF}$ power of $155 \mathrm{~W}$ and a chamber pressure of 50 mTorr. This process permits the fabrication, on both ends of the cavity, of $\sim 1$ - $\mu$ m-deep trenches with vertical and quite good flatness walls, that allow minimizing optical scattering losses. The two insets of Fig. 2 show both an optical and a scanning electron microphotograph (SEM) detail of the trenches.

\section{Experimental results and discussion}

We have tested the behaviour of the multistack device by performing DC measurements on the waveguide-integrated FP cavity.

A $\lambda=1550 \mathrm{~nm}$ light source from a $15 \mathrm{~mW}$ tunable laser-diode was fired into the device via a lensed fiber focused onto the input facet of the waveguide, and the output light was collected by a single-mode fiber and detected by an InGaAs photodiode.

For the $L=50-\mu \mathrm{m}$-long integrated cavity we measured, for different samples, a free spectral range (FSR) of $7.334 \pm$ 0.003 nm. By using [16]

$n_{g}(\lambda)=\frac{\lambda^{2}}{2 L \cdot F S R(\lambda)}$

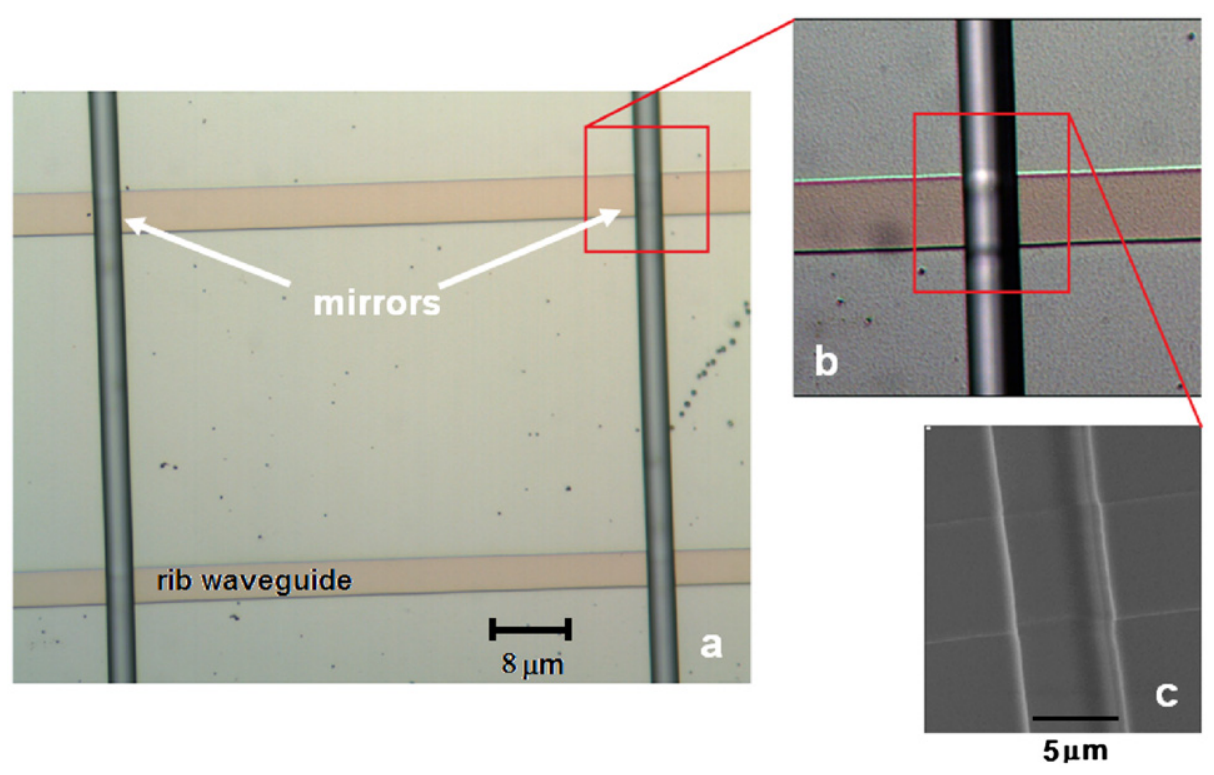

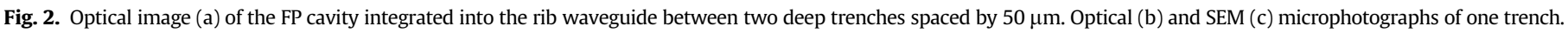


where $\lambda$ is the wavelength at each maximum of the transmission spectrum (resonance condition), a group index $n_{g}$ of $\sim 3.28$ could be calculated. From the same device, the extinction ratio of the cavity $I_{\max } / I_{\min }$, with $I_{\max }$ and $I_{\min }$ the maximum and minimum transmitted signals in a period of the spectrum respectively, was measured to be 1.39 .

The propagation loss introduced by the waveguide was calculated by using

$\alpha \cdot L=-\ln \left(\frac{1}{R} \cdot \frac{\sqrt{I_{\max } / I_{\min }}-1}{\sqrt{I_{\max } / I_{\min }}+1}\right)$

derived from the well known Airy's formula, where $R=\left(n_{\text {eff }}-n_{0}\right)^{2}$ / $\left(n_{\text {eff }}+n_{0}\right)^{2} \approx 0.28$ is the facet reflectance, with $n_{0}$ being the refractive index of air, and assuming $n_{\text {eff }} \sim n_{\text {g. }}$. In particular, we determined a value of $\alpha=20.7 \pm 0.5 \mathrm{~dB} / \mathrm{cm}$. However, the calculated value of $R \approx 0.28$ is only valid in the ideal case of perfectly smooth mirrors, thus the evaluated value for the propagation loss is indeed the worst case limit of the real losses introduced by the waveguide. The overall insertion losses for the 2.5-mm-long $(W=4 \mu \mathrm{m})$ device, including the $50-\mu \mathrm{m}$ Fabry-Perot cavity, were measured to be about $16 \mathrm{~dB}$. This rather high value is justified by the presence of the two 5 - $\mu \mathrm{m}$-wide air-trenches, and by the input/output rib waveguides facets, which did not receive polishing treatment in order to avoid resonance effects outside the cavity. Thus, for an optimized device, we plan to define shorter trenches by electron beam lithography (EBL) and improve the input/output coupling by taper and/or oblique facets.

The phase modulation measurements relied on the shift of the FP fringes when a bias was applied to the cavity region $(L=50 \mu \mathrm{m})$ between the $\mathrm{ZnO}$ :Al top contact and the grounded c-Si substrate. A DC voltage generator and electrical micro-probes were used for this purpose. Thus, the variation of the refractive index due to the applied voltage is directly evaluated from the change in the FP output interference pattern. In fact, the phase variation in the Airy's formula is given by

$\phi=\frac{2 \pi}{\lambda} n_{g} L$

When the refractive index changes, the same phase value occurs at all those wavelengths that satisfy the following:

$\Delta \phi=0=\frac{2 \pi L}{\lambda} \Delta n_{e f f}-\frac{2 \pi L}{\lambda^{2}} n_{g} \Delta \lambda$

By comparing the transmission spectra at two different biases, it is possible to measure $\Delta \lambda$ as the shift of the resonance wavelength, and therefore the electrically-induced change of the effective refractive index from Eq. (5), directly deriving from Eq. (4)

$\Delta n_{\text {eff }}(\lambda)=\frac{\Delta \lambda}{\lambda} n_{g}(\lambda)$

It is worth noting that, due to the presence of the insulating layers, no static power is dissipated during the measurements, so that no thermo-optic effect is induced.

As demonstrated in Ref. [10], the overall electro-absorption effect in a similar a-Si:H-based structure depends both on the optical (propagating light) and electrical (applied bias) field distributions within the a-SiCN/a-Si:H multistack waveguide core. In that work, the carriers profile under bias was estimated by means of electrical simulations and the derived results were used for optical simulations. The calculated bias-induced absorption coefficient was found to be in good agreement with the experimental data. Therefore, the phase shift of the optical beam due to a change of the modal effective index can be derived by Eq. (3), i.e.

$\Delta \phi=\frac{2 \pi}{\lambda} \Delta n_{e f f} L$ where $\Delta n_{\text {eff }}$ can be estimated analytically as well by considering an integral quantity as follows:

$\Delta n_{e f f} \cong \frac{\int_{0}^{t} \int_{0}^{w} \Delta n(x, y) I(x, y) d x d y}{\int_{0}^{t} \int_{0}^{w} I(x, y) d x d y}$

where $I(x, y)$ is the guided mode intensity distribution in each a-Si:H layer in the $\langle x, y\rangle$ plane orthogonal to the optical beam propagation direction, $\Delta n(x, y)$ is the local change in the refractive index induced by an inhomogeneous carriers profile when an external voltage $V$ is applied across the multistack device, $t$ is the device thickness, and $w$ is the active horizontal window where light propagates.

The fundamental mode profile has been calculated by the mode solver [13] showing that the optical beam is well confined under the rib of the waveguide (Fig. 4), with nearly 93\% of the TE polarization travelling beneath the rib. Thus we can safely take $w \approx W=4 \mu \mathrm{m}$ in Eq. (7).

Moreover, two-dimensional mixed electro-optical simulations were performed in the $\langle x, y\rangle$ plane in order to calculate numerically the effective refractive index change at different applied voltages. First, the device was electrically modelled using ATLAS, the device simulation package from Silvaco [17]. In particular, the simulation tool allows to simulate internal physics and device characterization of the device by numerically solving Poisson's equation and the charge continuity equations for free carriers. Moreover, we considered the carrier trapping effects and the releasing phenomena involving the shallow levels in the disordered a-Si:H films [18] by modeling the defects concentration typical of amorphous semiconductors [19,20]. As an example, Fig. 3 reports the calculated electron and hole concentration depth profile in the centre of one of the three insulator (a-SiC:H)-silicon, (a-SI:H)insulator, (a-SiC:H) structures forming the device.

Thus, for a bias of $11.5 \mathrm{~V}$ applied across the three bi-layer device, a carrier concentration of $\sim 10^{18} \mathrm{~cm}^{-3}$ was estimated close to the a-SiC:H/a-Si:H interface.

It is known that the refractive index change $(\Delta n)$ in silicon due to injection or depletion of free carriers can be derived to a first order approximation from the classical Drude model also for a-Si:H [21]:

$\Delta n=-\frac{e^{2} \lambda^{2}}{8 \pi^{2} c^{2} \varepsilon_{0} n}\left(\frac{\Delta N_{e}}{m_{e}}+\frac{\Delta N_{h}}{m_{h}}\right)$

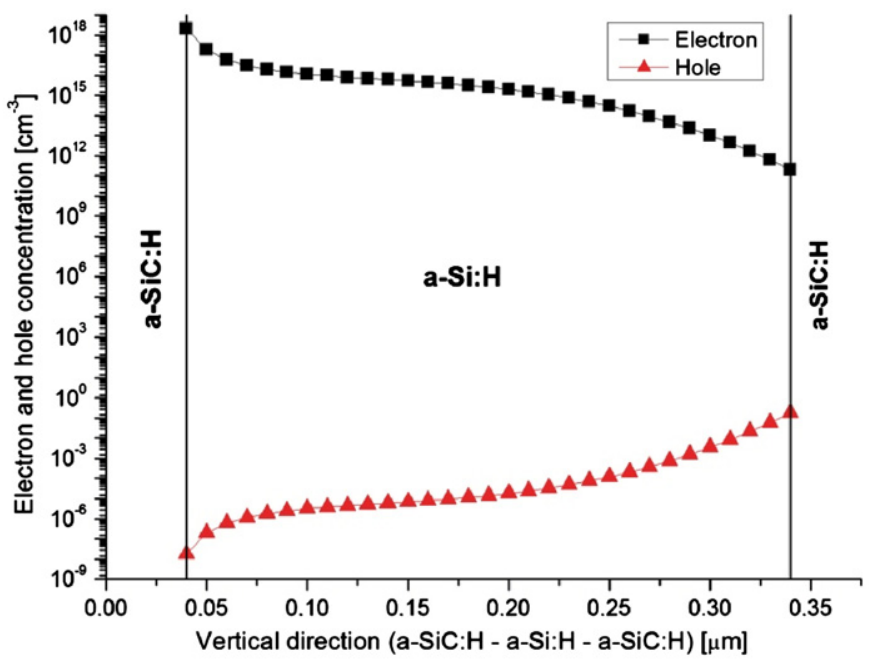

Fig. 3. Estimated electron and hole concentrations along a vertical section positioned at the center of the rib waveguide. The applied voltage is $11.5 \mathrm{~V}$ across the whole three bi-layers structure. 
a

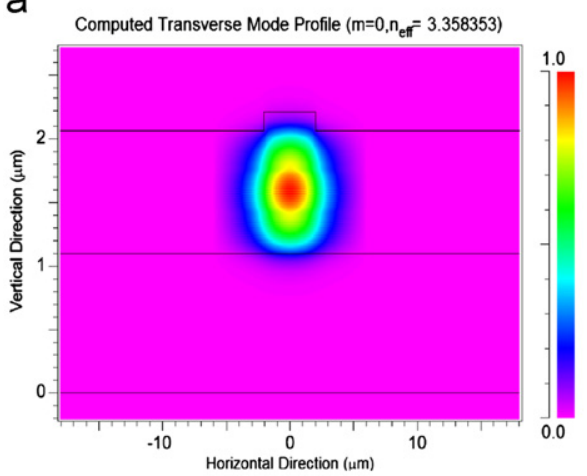

b

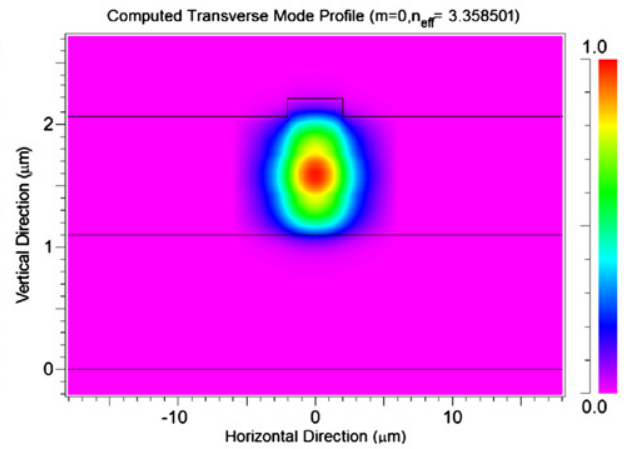

Fig. 4. Fundamental mode profiles, for TE polarization, respectively at $0 \mathrm{~V}$ (a) and $11.5 \mathrm{~V}$ (b). The calculated effective index variation is $\Delta n_{\text {eff }}=1.48 \times 10^{-4}$.

where $e$ is the electron charge, $\lambda$ is the optical beam wavelength, $\varepsilon_{0}$ is the permittivity of free space, and $m_{e}$ and $m_{h}$ are the effective masses of electrons and holes, respectively $\left(m_{e}=0.5 m_{0}, m_{h}=\right.$ $\left.1.0 m_{0}, m_{0}=9.1 \times 10^{-31} \mathrm{~kg}[21]\right)$.

Therefore, the newly calculated two-dimensional inhomogeneous refractive index profile, at several bias points, was fed into the optical simulator in order to evaluate the free-carrier dispersion effect on the optical propagation. In particular, a uniform grid size $(1 \mathrm{~nm}$ along the vertical axis and $10 \mathrm{~nm}$ along the horizontal direction) was employed for both electrical and optical simulations. In Fig. 4 we report the TE fundamental mode together with the modal effective index values, respectively at $0 \mathrm{~V}$ (Fig. $4 \mathrm{a}$ ) and $11.5 \mathrm{~V}$ (Fig. 4b), from which we calculated an effective index variation, $\Delta n_{\text {eff }}$, of $1.48 \times 10^{-4}$.

In our experiments, the measured $\Delta n_{\text {eff }}$ increases with the bias signal as shown in Fig. 5 because more and more carriers are accumulated at the opposite sides of each insulating film [5], thus leading to a large interaction between the optical beam and the accumulation layers. In fact, the thin a-SiC:H insulating layers break the conduction among the a-Si:H films so that the device effectively behaves as a series of capacitors. We observed, the occurrence of breakdown phenomena, most probably in the a-SiC:H dielectric layers, for applied voltages higher than $\sim 12-13 \mathrm{~V}$, thereby limiting the field-induced effects. By comparing the optical spectra of the cavity at zero bias and at $11.5 \mathrm{~V}$, we calculated a red shift of the spectrum of $\Delta \lambda \sim 0.071 \mathrm{~nm}$, from which the induced $\Delta n_{\text {eff }}$ (Eq. (5)) of the guided mode was estimated to be $1.51 \times 10^{-4}$ in very good agreement with simulation results.

To evaluate the phase modulation efficiency, a figure of merit is usually adopted, defined as the product $V_{\pi} \times L_{\pi}$, where $V_{\pi}$ and $L_{\pi}$ are respectively the bias voltage and the device length required to obtain a phase shift of $\pi$ of the guided optical wave. The smaller this product, the more efficient will the modulator be. With the measured $\Delta n_{\text {eff }}$, we get $V \pi \times L \pi=5.9 \mathrm{~V} \mathrm{~cm}$, not far from what was observed in high performing electro-optical modulators in crystalline silicon $[1,2,6,16]$.

As already discussed in Ref. [5], we expect that the dynamic behaviour of the device limits its application to integrated spatial light switches, e.g. digital optical switches (DOS) [22]. This limit comes from the characteristic times of the carriers trapping and releasing phenomena involving the shallow levels in the disordered a-Si:H films [20,23]. In fact, each time a bias is applied, a transient takes place during which the already existing carriers are swept toward the semiconductor/insulator interfaces, together however with many other carriers, newly generated with their own characteristic time constant. The same applies at bias removal, when evolution of the carrier population is largely dominated by recombination. This is similar to what happens in

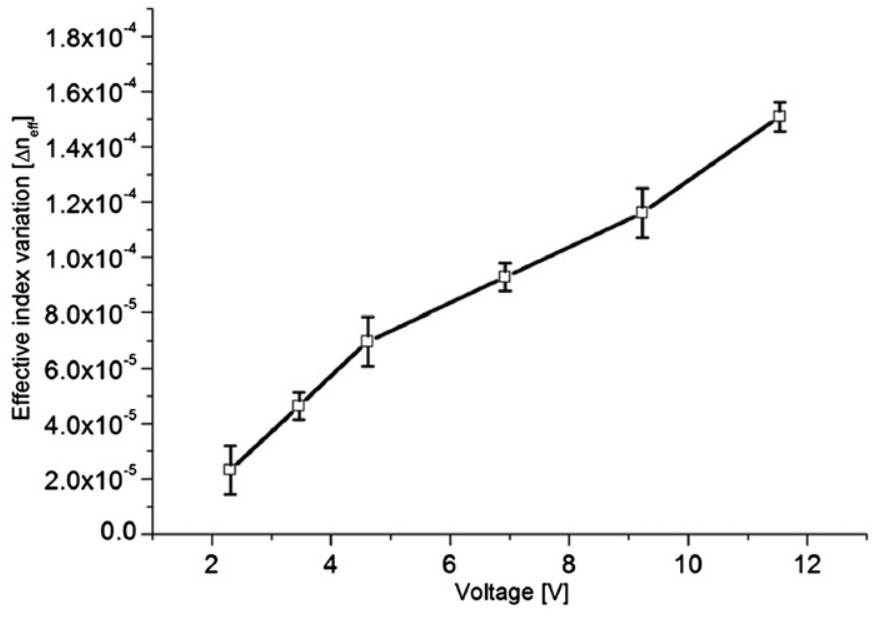

Fig. 5. Experimental effective index variation vs. bias voltage ranging from 0 to $11.5 \mathrm{~V}$. The error bars refer to different measurements on $L=50-\mu \mathrm{m}$-long integrated FP cavities.

the channel of a-Si:H TFT's, for which cut-off frequencies of $1 \mathrm{MHz}$ have been reported [24].

\section{Conclusion}

We demonstrated that effective electro-optical phase shift at $\lambda=1.55 \mu \mathrm{m}$ can be produced in a multistack waveguiding structure based on the CMOS-compatible technology of a-Si:H. The introduction of insulating layers in the waveguide core results in an enhancement of the free carrier accumulation effect at the multiple a-Si:H/a-SiC:H interfaces inducing, therefore, a higheffective index variation. The structure allows to achieve a refractive index variation due to free carrier variation in the waveguide core of $1.51 \times 10^{-4}$ at $11.5 \mathrm{~V}$, in good agreement with mixed electro-optical simulation results. As a consequence, the voltage-length product for inducing a phase variation $\Delta \phi=\pi$ in an optical travelling wave was determined to be $V_{\pi} \times L_{\pi}=5.9 \mathrm{~V} \mathrm{~cm}$, not far from what was observed in high performing electro-optical modulators in crystalline silicon.

\section{Acknowledgements}

This work was partially developed under the Seventh Framework Programme (FP7/2007-2013) of the European Union, IST project "HELIOS"-Grant Agreement Number 224312. 


\section{References}

[1] Green WMJ, Rooks MJ, Vlasov LSc, and YA. Ultra-compact, low RF power, $10 \mathrm{~Gb} / \mathrm{s}$ silicon Mach-Zehnder modulator. Optics Express 2007;15(25): 17106-13.

[2] Liu A, Liao L, Rubin D, Nguyen H, Ciftcioglu B, Chetrit Y, Izhaky N, Paniccia M. High-speed optical modulation based on carrier depletion in a silicon waveguide. Optics Express 2007;15(2):660-8.

[3] Narayanan K, Elshaari AW, Stefan FPreble. Broadband all-optical modulation in hydrogenated-amorphous silicon waveguides. Optics Express 2010;18(10):9809-14.

[4] Selvaraja SK, Sleeckx E, Schaekers M, Bogaerts W, Van Thourhout D, Dumon P, Baets R. Low-loss amorphous silicon-on-insulator technology for photonic integrated circuitry. Optics Community 2009;282:1767-70.

[5] Della Corte FG, Rao S, Nigro MA, Suriano F, Summonte C. Electro-optically induced absorption in a-Si:H/a-SiCN waveguiding multistacks. Optics Express 2008;16(10):7540-50.

[6] Preston K, Manipatruni S, Gondarenko A, Poitras CB, Lipson M. Deposited silicon high-speed integrated electro-optic modulator,. Optics Express 2009;17(7):5118-24.

[7] Della Corte FG, Rao S, Coppola G, Summonte C. Electro-optical modulation at $1550 \mathrm{~nm}$ in an as-deposited hydrogenated amorphous silicon $\mathrm{p}-\mathrm{i}-\mathrm{n}$ waveguiding device. Optics Express 2011;19(4):2941-51.

[8] Cocorullo G, Della Corte FG, De Rosa R, Rendina I, Rubino A, Terzini E. Amorphous silicon-based guided-wave passive and active devices for silicon integrated optoelectronics. IEEE Journal of Selected Topics in Quantum Electronics 1998;4(6):997-1002.

[9] Barrios CA. Electrooptic modulation of multisilicon-on-insulator photonic wires,. Journal of Lightwave Technology 2006;24:2146-55.

[10] Rao S, Della Corte FG, Summonte C, Suriano F. Electro-optical modulating device based on a CMOS-compatible a-Si:H/a-SiCN multistack waveguide. IEEE Journal of Selected Topics in Quantum Electronics 2010;16(1):173-8.

[11] Zelikson M, Salzman J, Weiser K, Kanicki J. Enhanced electro-optic effect in amorphous hydrogenated silicon based waveguides.. Applied Physics Letters 1992;61(14):1664-6.
[12] Rao S., Coppola G., Gioffrè M., Summonte C., Della Corte F.G.. Characterization of an electrically induced refractive index change in a hydrogenated amorphous silicon multistack waveguide. Proceedings of 8th IEEE international conference on group IV photonics (GFP); 2011, p. 302-4.

[13] R Soft Photonics CAD Layout User Guide, R soft Design Group, Inc. Physical Layer Division, 200 Executive Blvd. Ossining, NY 10562.

[14] Barrios CA, Lipson M. Modeling and analysis of high-speed electro-optic modulation in high confinement silicon waveguides using metal-oxidesemiconductor configuration,. Journal of Applied Physics 2004;96(11): 6008-15.

[15] Liu A, Jones R, Liao L, Samara-Rubio D, Rubin D, Cohen O, Nicolaescu R, Paniccia M. A high speed silicon optical modulator based on a metal-oxidesemiconductor capacitor. Nature 2004;427:615-8.

[16] Marris-Morini D, Le Roux X, Vivien L, Cassan E, Pascal D, Halbwax M, Maine S, Laval S, Optical modulation by carrier depletion in a silicon PIN diode, Optics Express 2006;14:10838-43.

[17] ATLAS device simulation software user's manual Santa Clara, CA: SILVACO International.

[18] Street RA. Hydrogenated amorphous silicon. Cambridge, UK: Cambridge University Press; 1991.

[19] Singh J. Effective mass of charge carriers in amorphous semiconductors and its applications,. Journal of Non-Crystalline Solids 1973;120:295-300.

[20] Della Corte FG, Rubino A, Cocorullo G. Simulation study and realisation of an $\alpha$-Si:H emitter on GaAs,. Solid-State Electronics 1998;42:1819-25.

[21] Fauchet PM, Hulin D, Vanderhaghen R, Mourchid A, Nighan Jr. WL. The properties of free carriers in amorphous silicon. Journal of Non-Crystalline Solids 1992;141:76-87.

[22] Iodice M, Mazzi G, Sirleto L. Thermo-optical static and dynamic analysis of a digital optical switch based on amorphous silicon waveguide,. Optics Express 2006;14(12):5266-78.

[23] Singh J. Effective mass of charge carriers in amorphous semiconductors and its applications. Journal of Non-Crystalline Solids 2002;299-302(1):444-8.

[24] Fukuda K, Imai N, Kavamura S, Matsumura K, Ibaraki N. Switching performance of high rate deposition processing a-Si:H TFTs. Journal of NonCrystalline Solids 1996;198-200:1137-40. 